\title{
NEIL3-mediated mitotic base excision repair of oxidative lesions at telomeres prevents senescence in hepatocellular carcinoma
}

Helge Gad'1,3*, Zhao Zhenjun'2, Carlos Benitez-Buelga', Kumar Sanjiv', Hua Xiangwei', He Kang'2, Feng Mingxuan², Zhao Zhicong'2, Ulrika Warpman Berglund', Xia Qiang'2, Thomas Helleday ${ }^{1,3}$

1) Science for Life Laboratory, Department of Oncology-Pathology, Karolinska Institutet, S-171 76 Stockholm, Sweden. 2) Department of Liver Surgery, Renji Hospital, School of Medicine, Shanghai Jiao Tong University, Shanghai, China.

3) Weston Park Cancer Centre, Department of Oncology and Metabolism, University of Sheffield, Shefffild S10 2RX, UK. 4) Organ Transplantation Center, the Afflilated Hospital of Qingdao University, Qingdao, China

Introduction

Hepatocellular carcinoma (HCC) is the most common type of primary liver cancer and the third leading cause of cancer death worldwide. Chronic liver diseases contributes to accumulation of reactive oxygen species (ROS) and inflammation, leading to cirrhosis and eventually HCC. Although many approaches have been suggested to treat $\mathrm{HCC}$ (e.g., surgery, transarterial chemoembolization (TACE), immunotherapy and targeted therapies), effective drugs and non-surgical treatment for HCC patients remain very limited

ROS production increases during G2/M-phase and induction of oxidative damage in vells in pro-metaphase, suggesting that the level of endogenous DNA damage varies during the cell cycle and have more severe effects in certain phases.

It is well established that there is differential DNA repair at telomeres. Telomeres are sensitive to oxidative damage, resulting in cell senescence, chromosome fusion and apoptosis. While BER pathways are described to be active at telomeres, information about these processes or potential differential roles of glycosylases are generally lack-

(1) NEIL3 is overexpressed in HCC which correlates
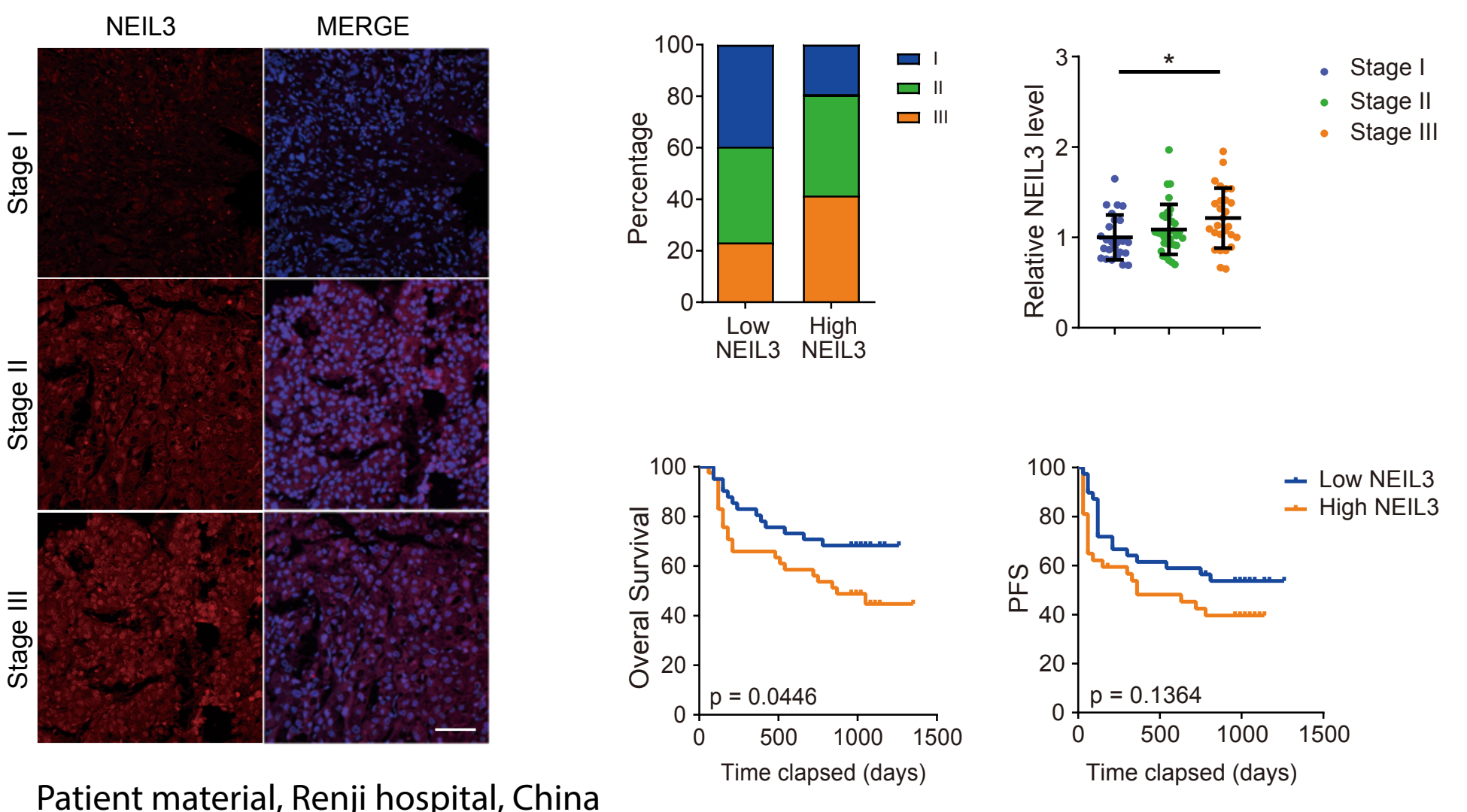

2 HCC cell lines require NEIL3 for cell proliferation in vitro
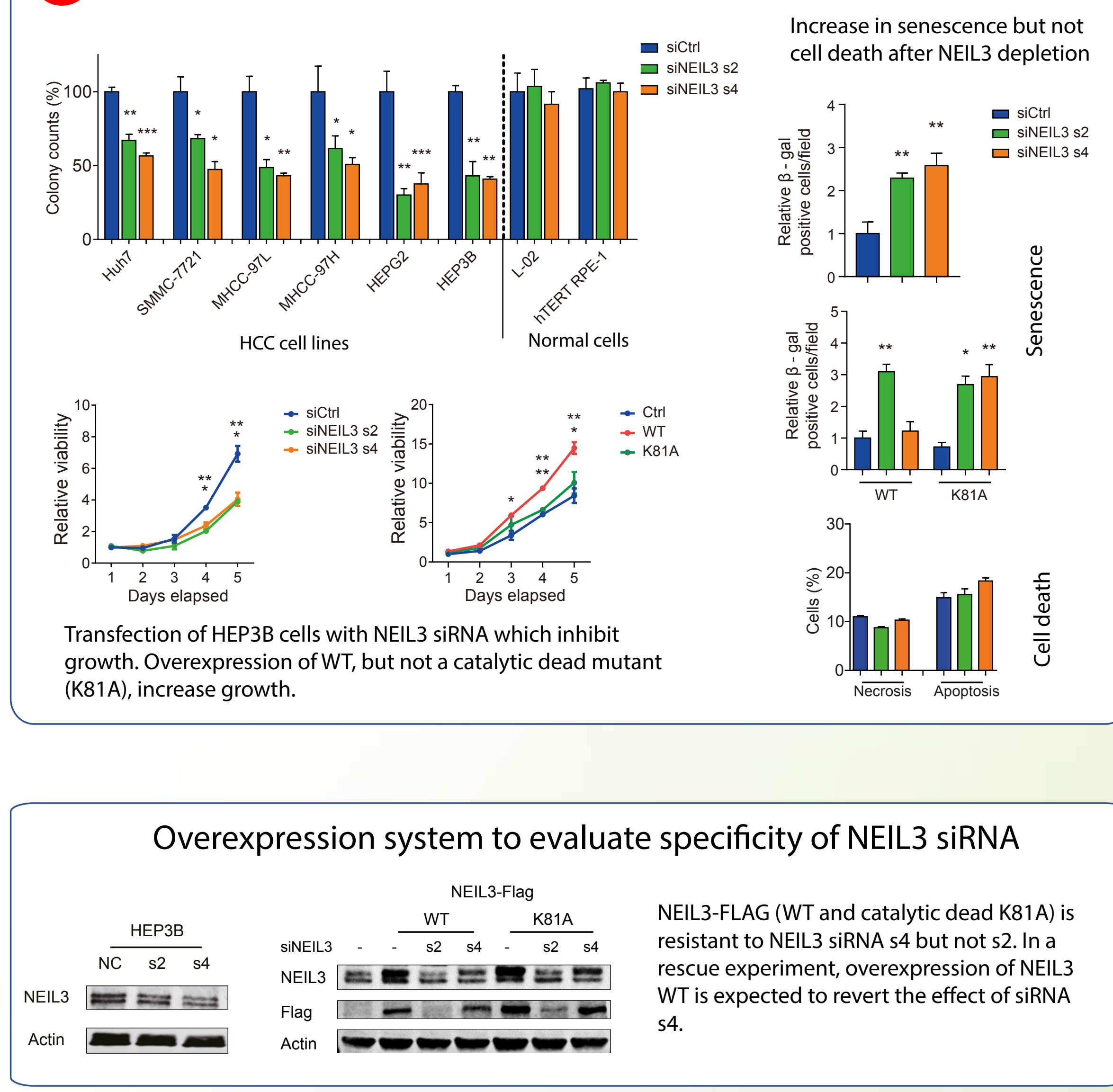

NEIL3 silencing induces oxidative damage at telomeres

(3) Modified comet assay with Endo VIII digestion
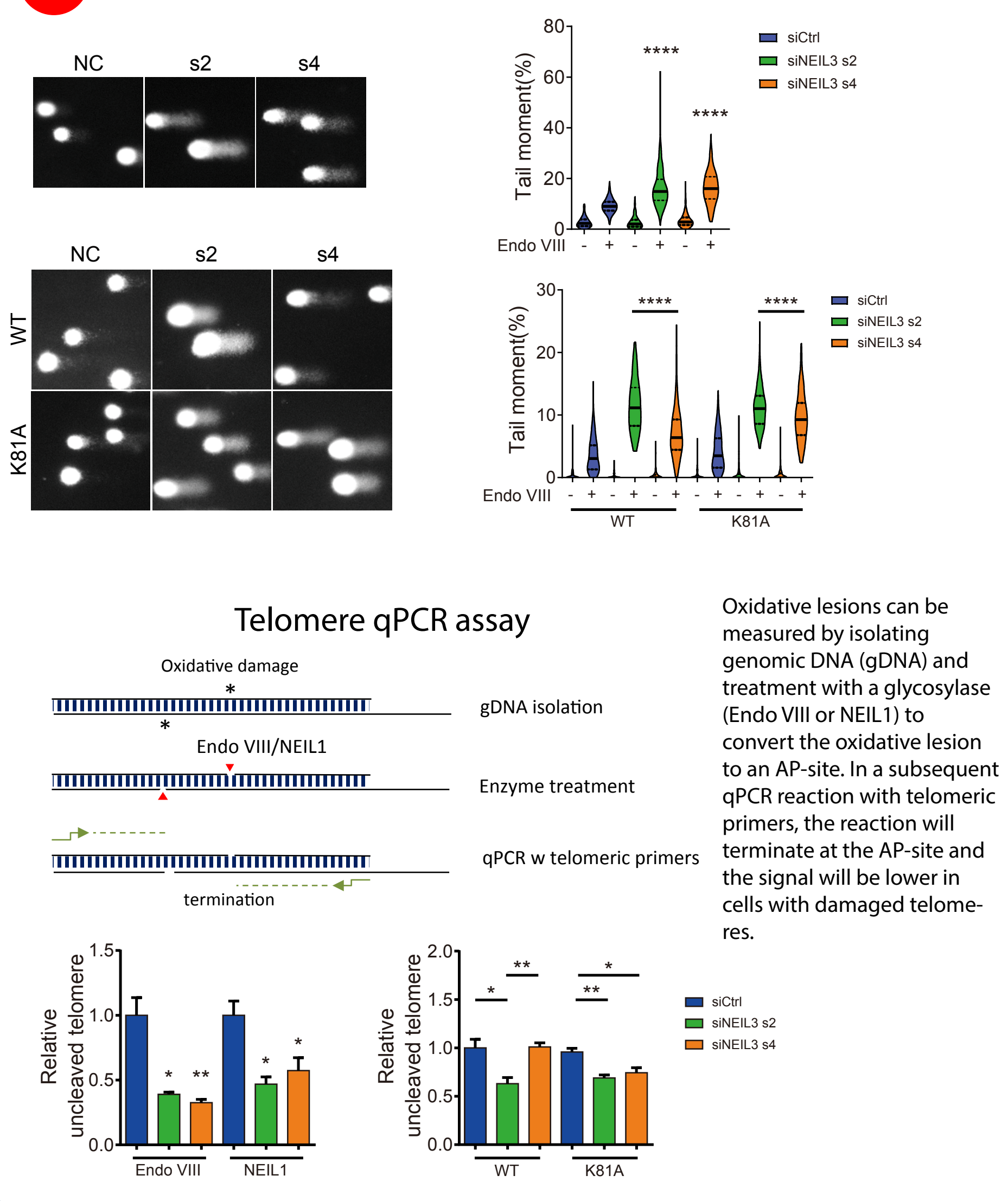

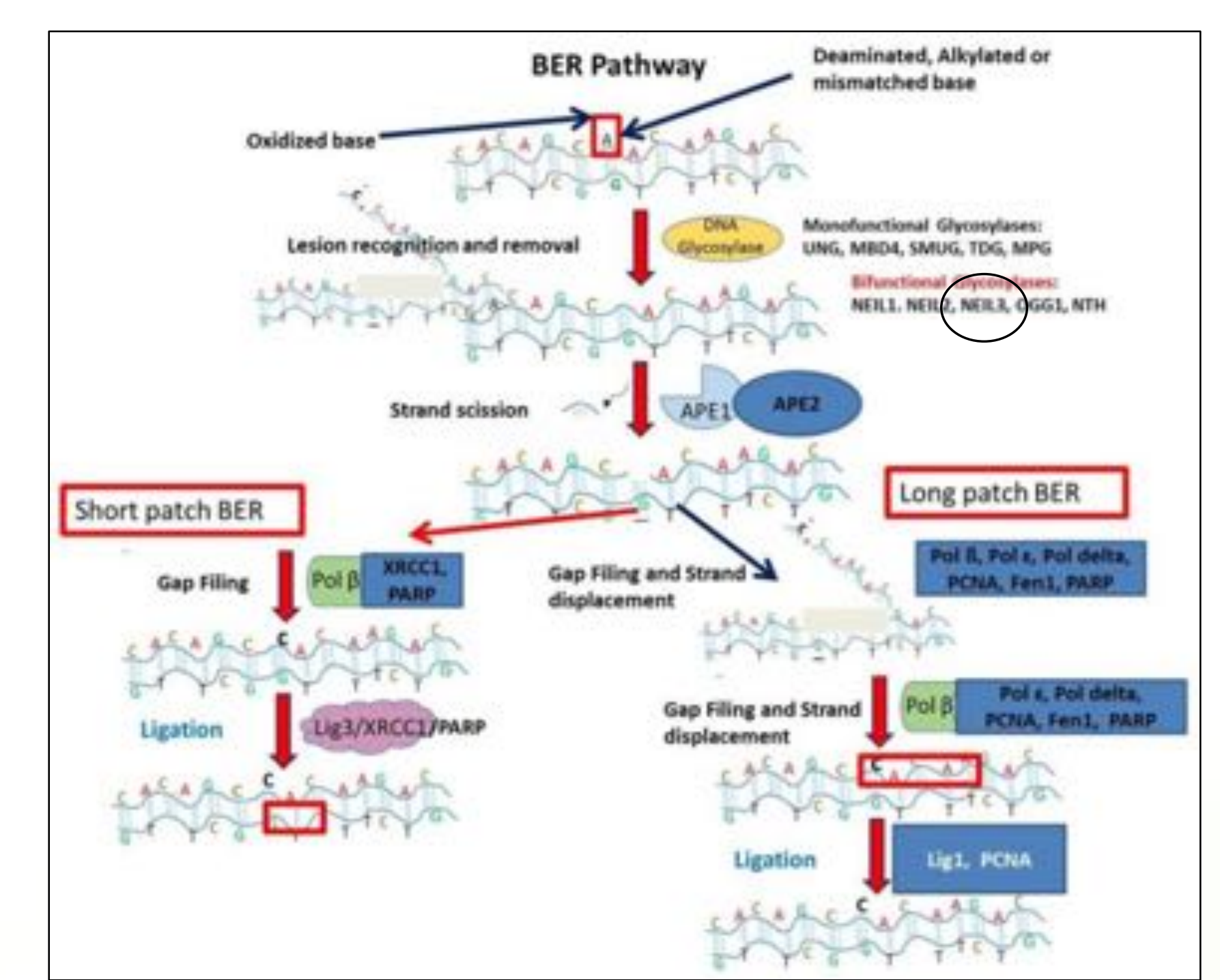

Oxidative damaged bases in DNA are repaired by Base Excision Repair (BER). NELLZ is a multi-
functional glycosylase removing hydantoins (spiroiminodihydantoin (Sp) and guanididiohydan-

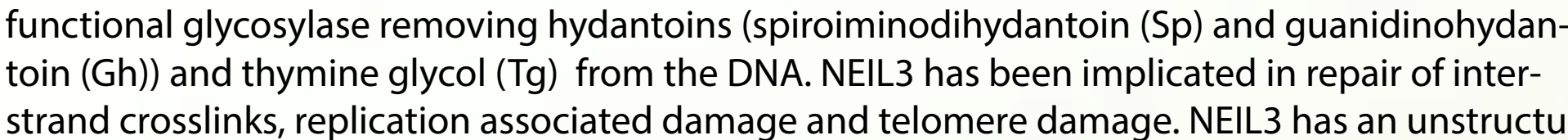
strand crosslinks, replication associated damage and telomere damage. NELL 1 ,
red C-terminal domain that can interact with TRF1, FEN-1, PCNA and APE-1.

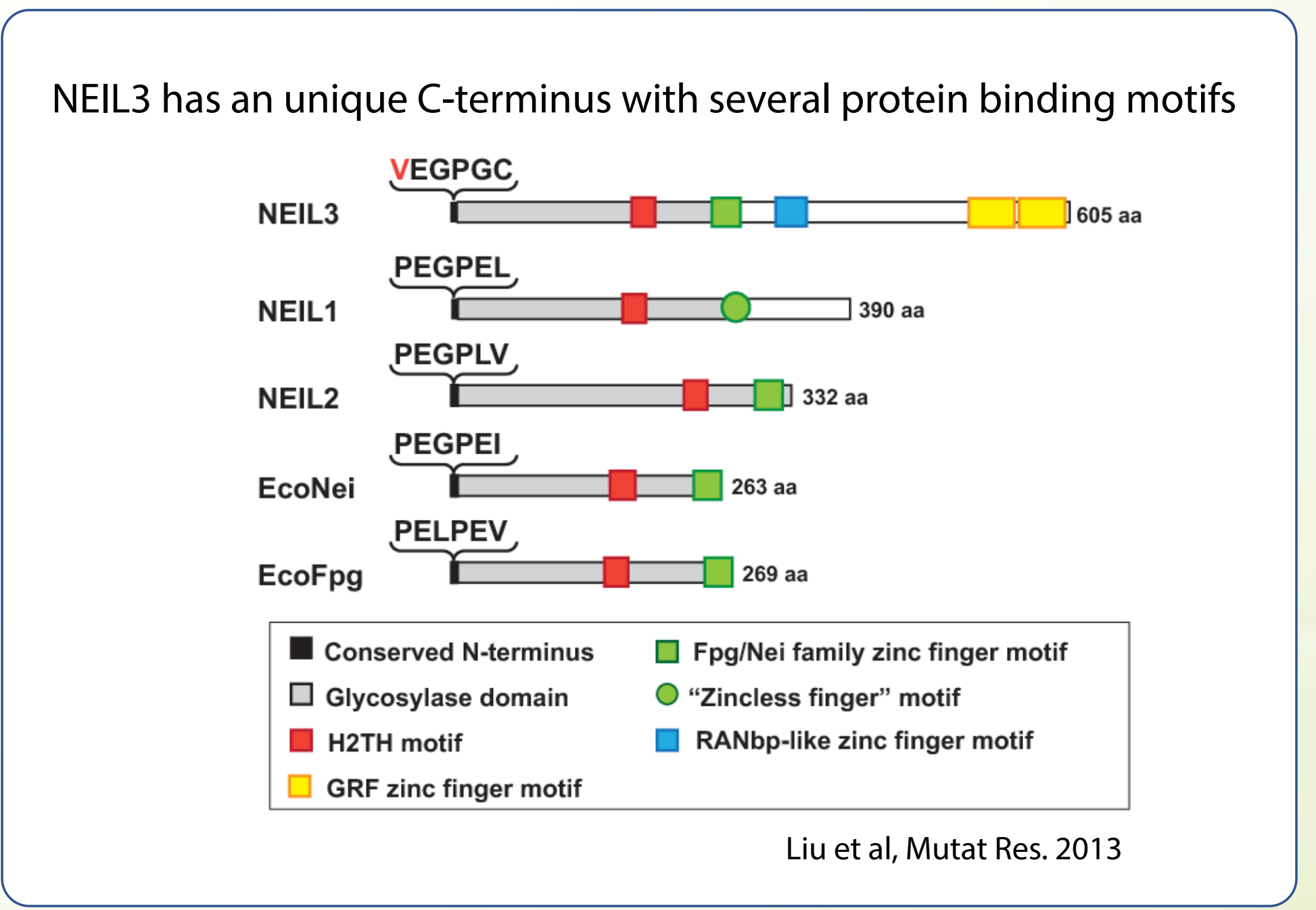

The increase of NEIL3 expression in G2/M phase and the observation that oxidative stress has profound effects in mitosis,
NEIL3 has a crucial role in DNA repair in this phase.

6
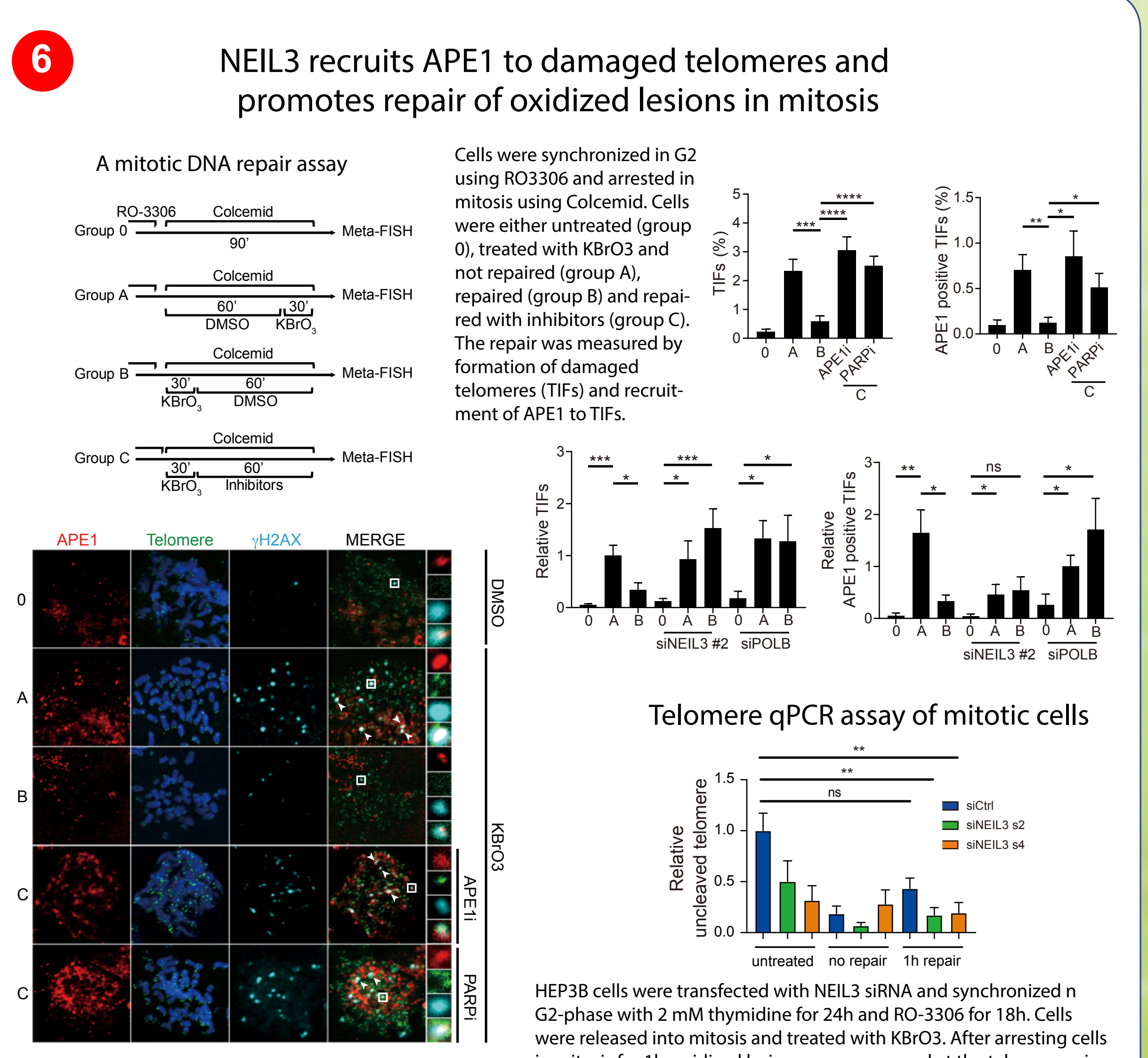

Telomere qPCR assay of mitotic cells
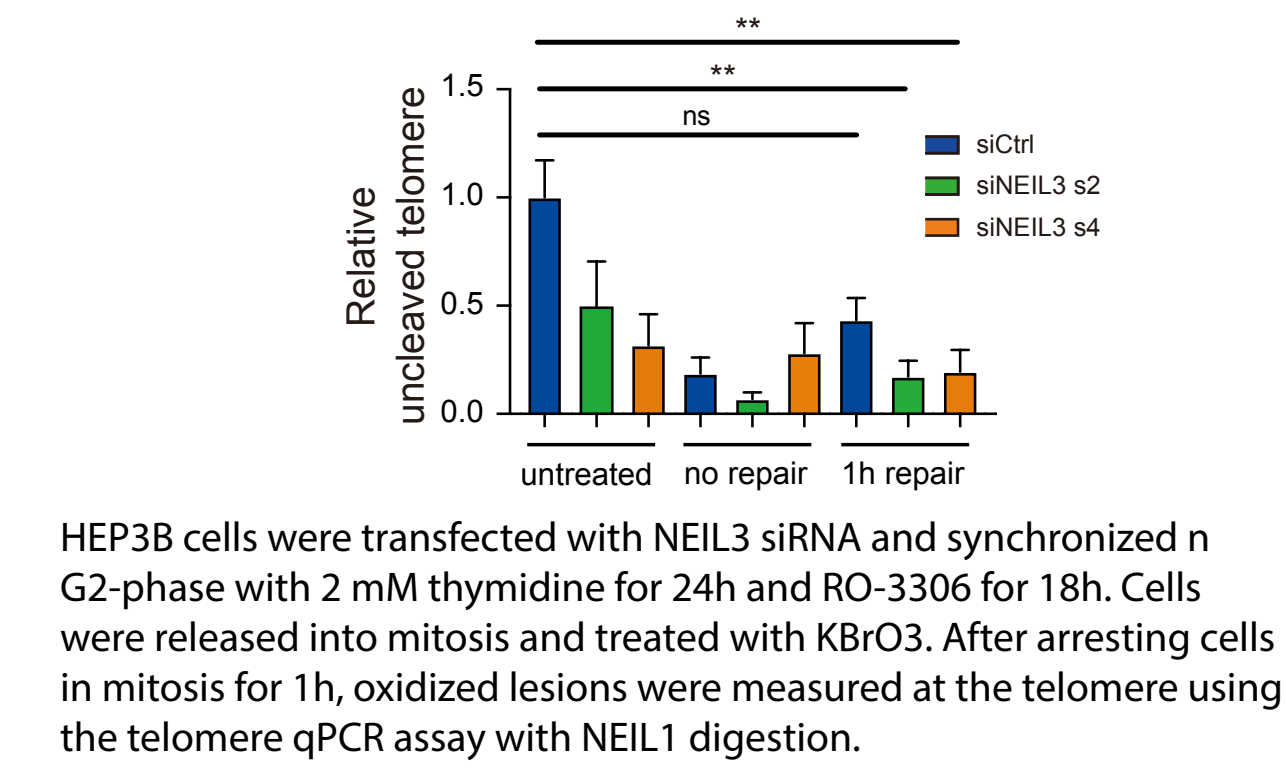

7 NEIL3 protects HEP3B cells from genomic instability induced by
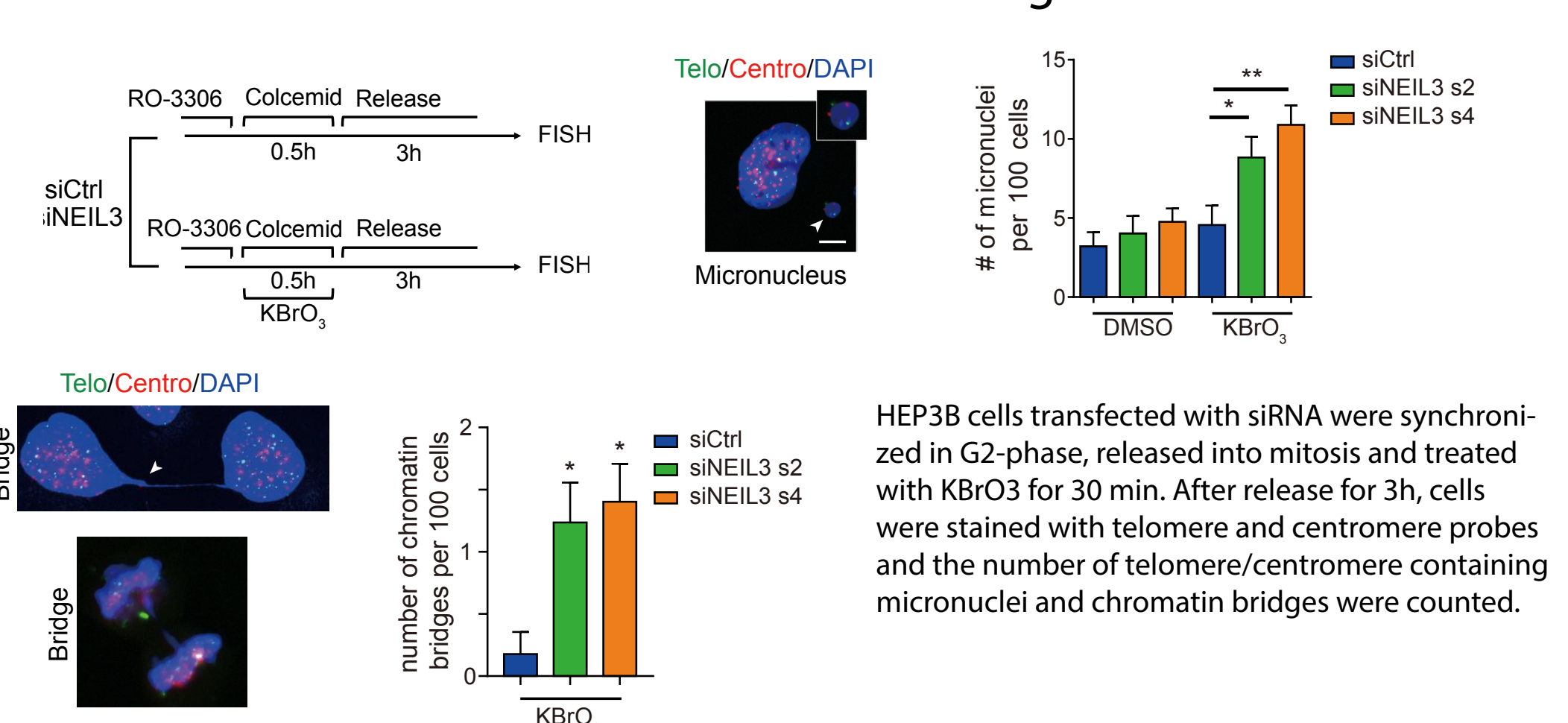

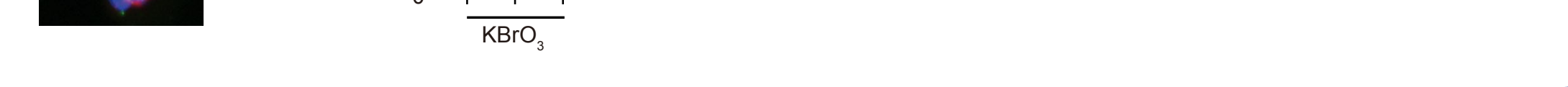

Summary

EEL 3 is overexpressed in HCC which correlates with poor survival.

EEIL3 depleted HCC cell lines accumulate oxidative DNA lesions specifically at telomeres, resul-

NEL3 relocates to telomeres following oxidative DNA damage during mitosis and recruits apuri(APE1), indicating activation of base excision repair.

NELL3, but not NELL1 or NELL2, is required to initiate base excision repair at oxidized telomeres and damaged telomeres.

These data suggest NEIL 3 could be a target for therapeutic intervention of HCC, and perhaps a combination treatment with a NEIL3 inhibitor and oxidizing compounds (e.g., elesclomol) could prove to be a good strategy to induce ROS and prevent its repair in the tumour.

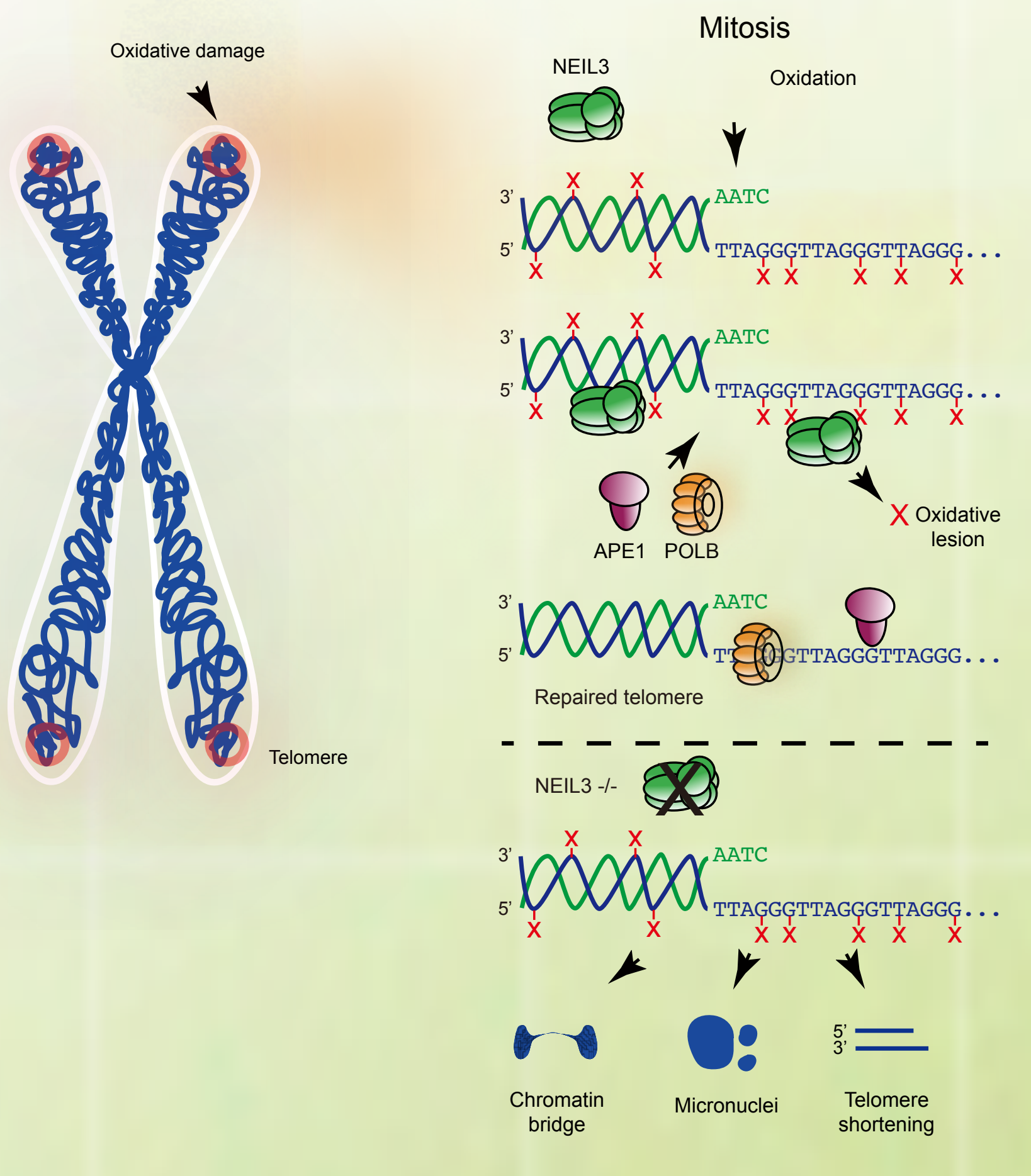

(4. NELL3 is localized to damaged telomeres induced by

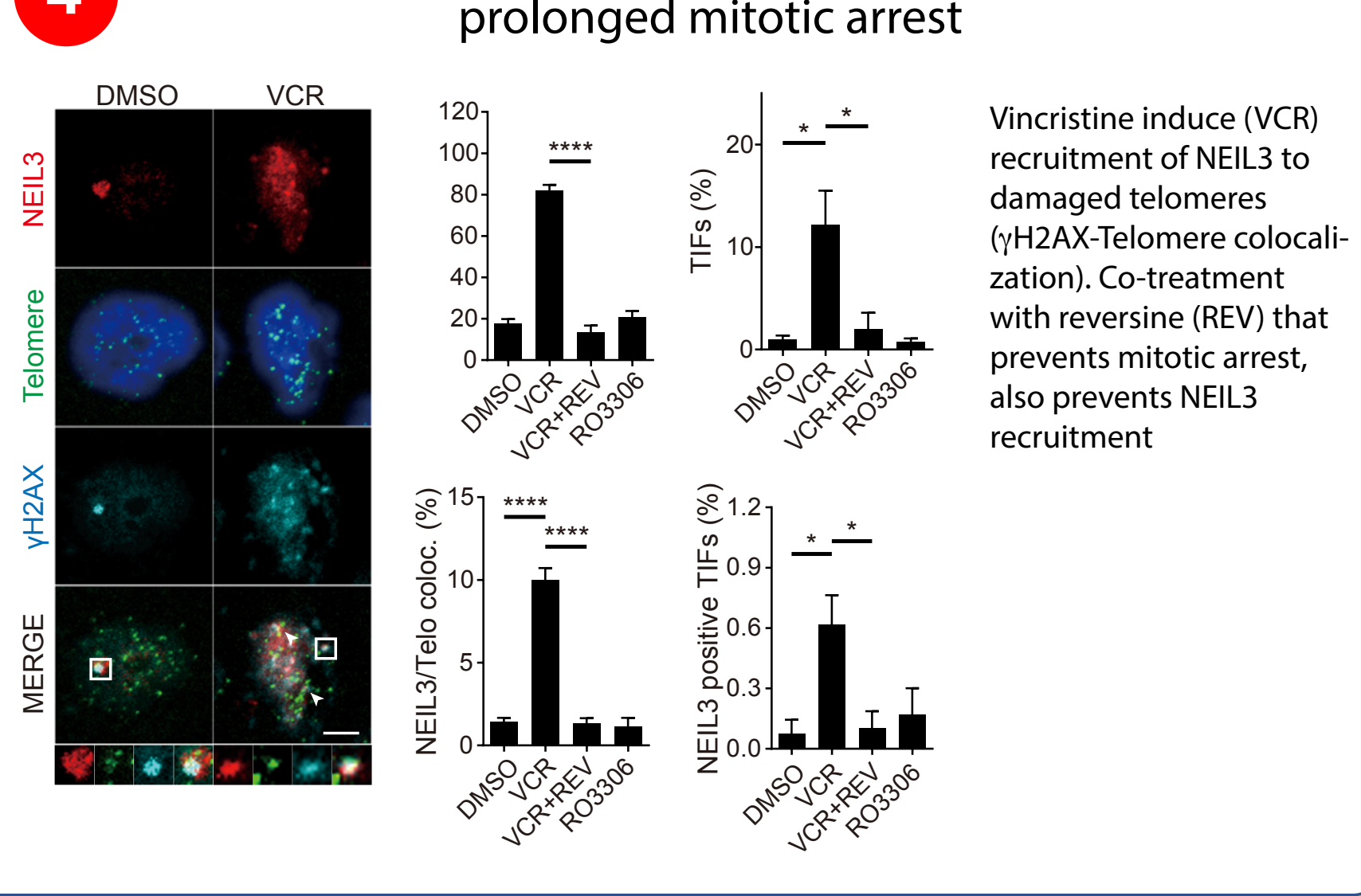

5 NEIL3, but not NEIL1 and 2, are required for recruitment

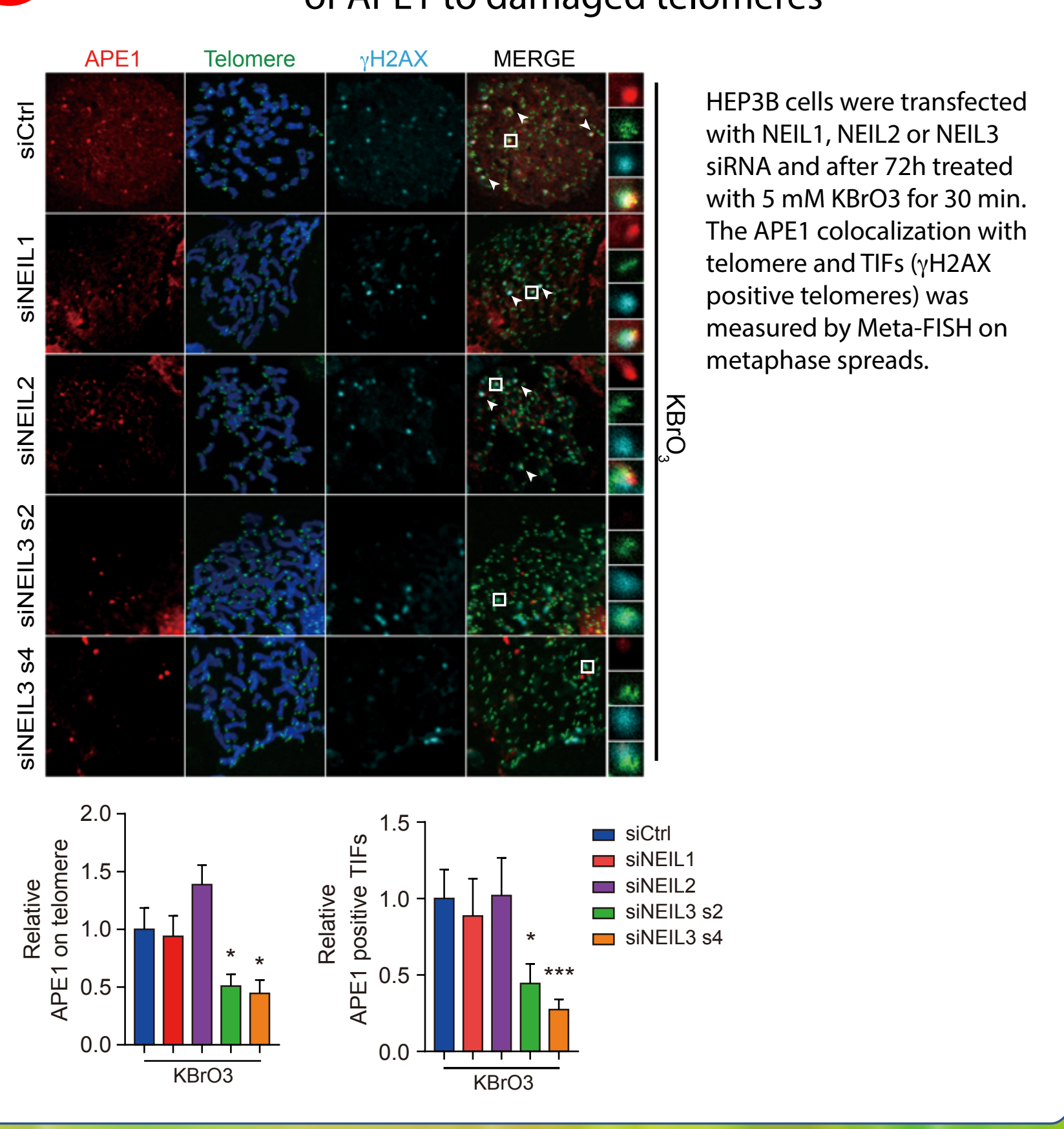

Overexpression of NEIL3 protects cells from long-term effects of 8

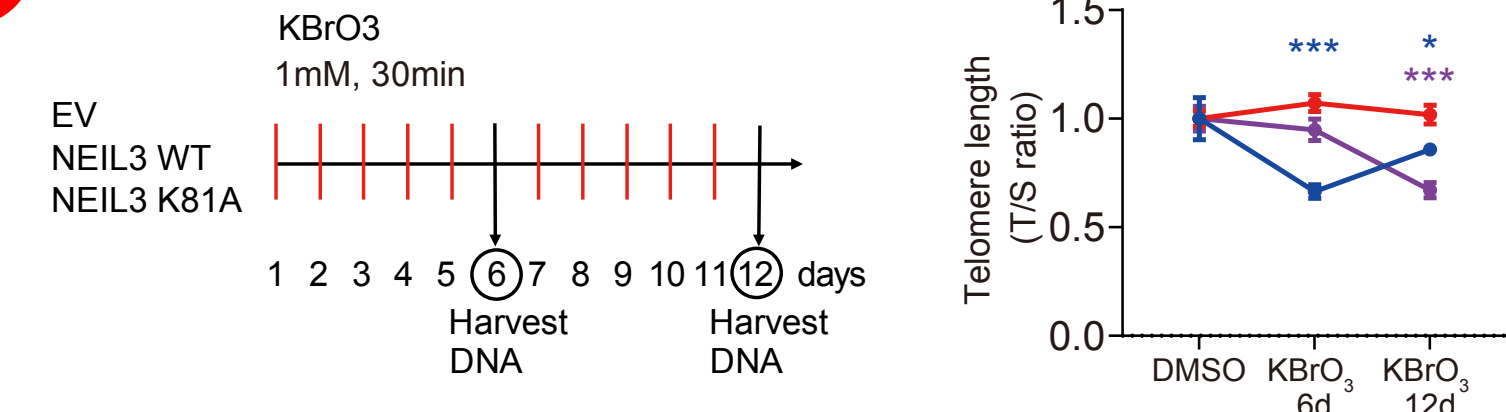

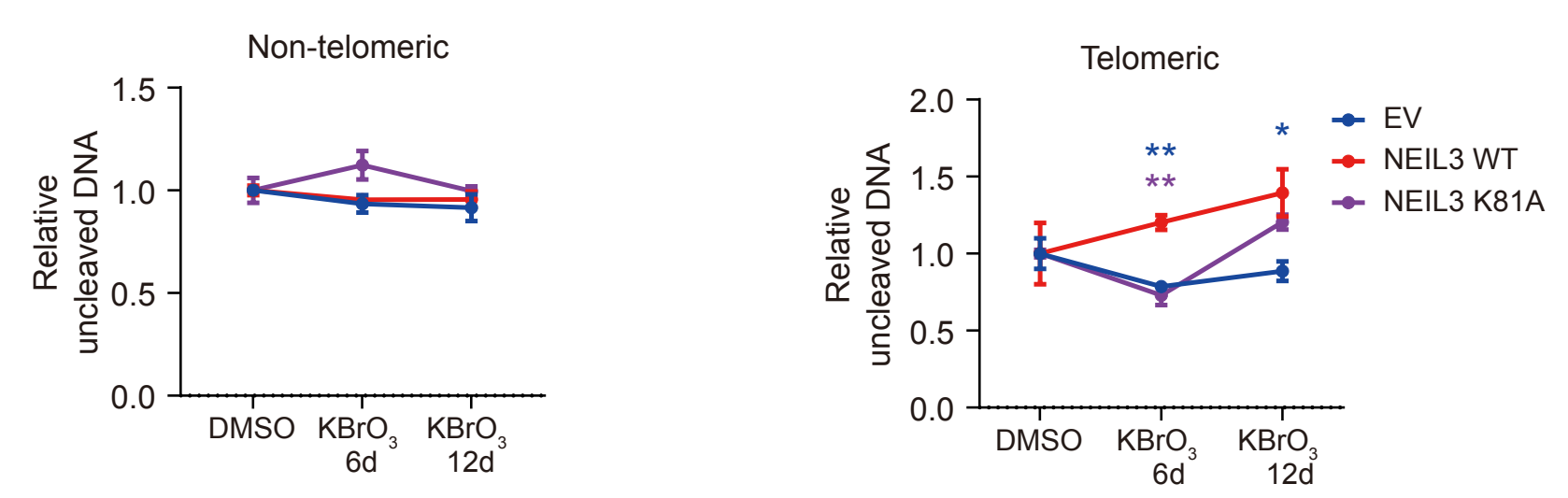

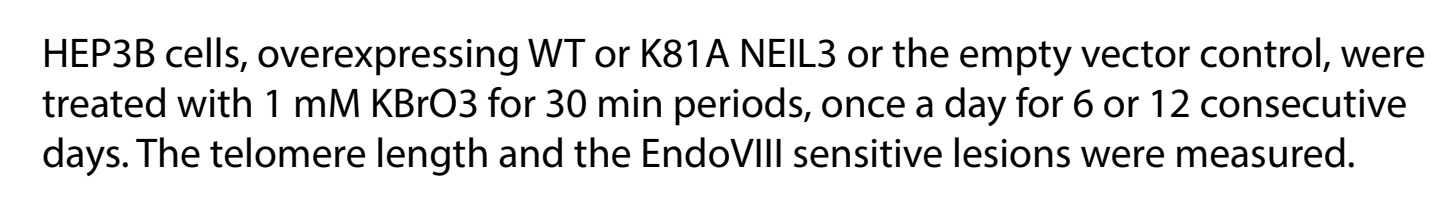

